EPJ Web of Conferences 60, 20009 (2013)

DOI: $10.1051 /$ epjconf $/ 20136020009$

(C) Owned by the authors, published by EDP Sciences, 2013

\title{
Investigation of phenomenological models implemented in PYTHIA6
}

\author{
Nameeqa Firdous ${ }^{1, a}$, Gerald Rudolph ${ }^{1}$ \\ ${ }^{1}$ Institute of Astro and Particle Physics, University of Innsbruck, Austria
}

\begin{abstract}
Different phenomenological models implemented in PYTHIA6 using best fit to Minimum Bias (MB) data published by ATLAS experiment at two center of mass energies $0.9 \mathrm{TeV}$ and $7 \mathrm{TeV}$ are investigated.
\end{abstract}

\section{Introduction}

PYTHIA is highly successful and well established Monte Carlo event generator and developed over the past decades with the experimental discoveries. It can describe most of the features observed in past and present experiments. The core of PYTHIA's model for soft hadronic interactions is based on a phenomenological adaptation of QCD to describe the non perturbative pp processes [1]. In this work we studied different Physics models given by PYTHIA6 to describe their behavior at high energies and to improve our preliminary tunes [6]. PYTHIA6 offers variety of Physics models which can be selected with the so called switches. Each model is defined by certain parameters which are mostly free and need to be tuned. We investigated selected models by tuning their free parameters using ATLAS Minimum Bias (MB) published data [3] at two center of mass energies, 0.9 and $7 \mathrm{TeV}$. The model combination investigated are shown in Figure 1. The choice of models as well as their combinations are totally arbitrary.

\section{MPI Model}

Since the proton or any other hadron is a composite object, in a head on collision of two hadron there is a probability that more than one pair of partons undergo scatterings, resulting in a higher multiplicity compared to events with only one strong interaction. PYTHIA6 offer this model with the switch $\operatorname{MSTP}(81)$.

- For $\operatorname{MSTP}(81)=0$, MPI is switched off.

- For MSTP(81)=21, MPI is switched on with new scenario of multiple parton interactions, interleaved with ISR, and pt as ordering variable

Studies of the average particle transverse momentum pT as a function of the events charged particle multiplicity Nch, Figure 2, demonstrates that models fail to describe the data if the possibility of multiple parton interactions is neglected. This observable is sensitive to the presence of MPI since a higher multiplicity, which is a result of MPI,

\footnotetext{
ae-mail: nameeqa@yahoo.com
}

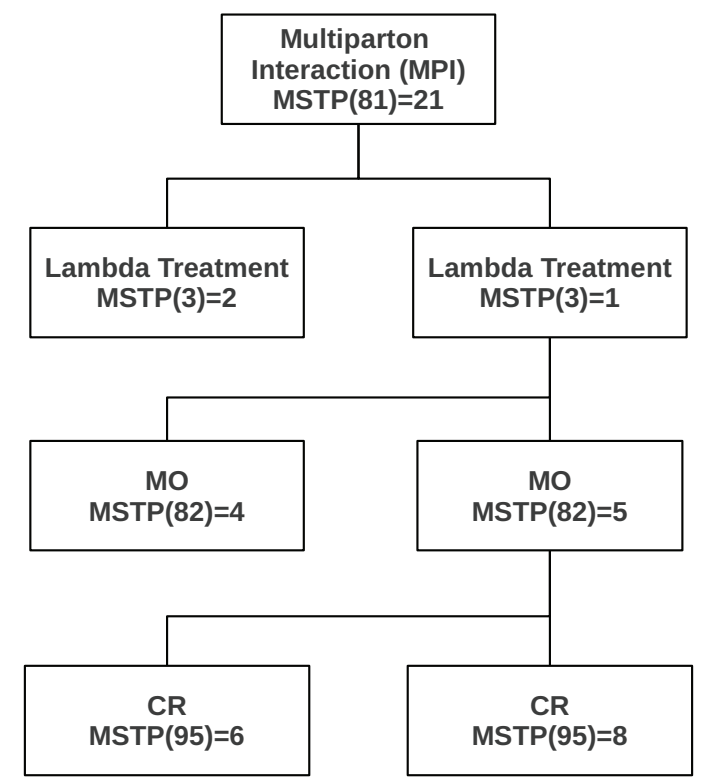

Figure 1. Flow diagram showing selected combination of different models implemented in PYTHIA6 framework.

result in a lower average pT. In the absence of MPI the average $\mathrm{pT}$ shows harder spectrum. The low activity can also be seen in the multiplicity distribution. If MPI is switched on the model describes the data convincingly well.

\section{Lambda Treatment}

PYTHIA6 provides possibilities to select the same $\Lambda_{Q C D}$ in the definition of alpha evolution for ISR, ME and FSR as well as three different values. The switch MSTP(3) gives the control over the selection of lambda values.

- For $\operatorname{MSTP}(3)=2, \Lambda_{Q C D}$ value is chosen according to the parton distribution function parameterizations. Then 


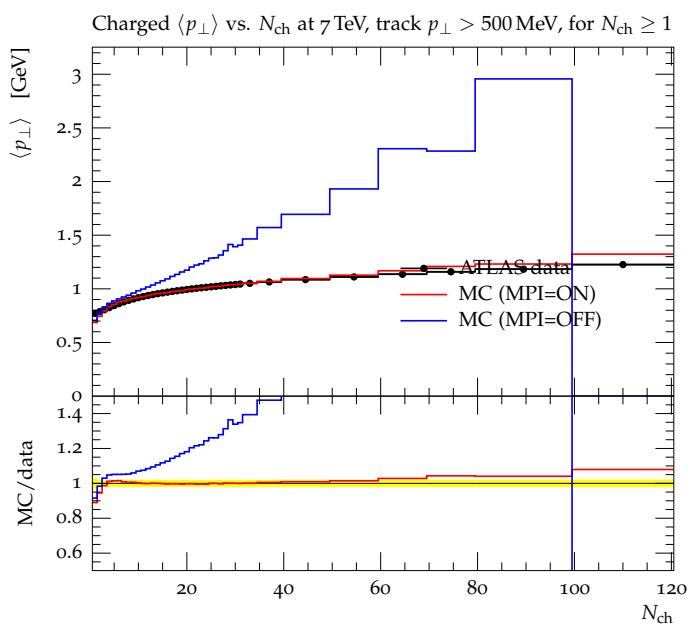

Figure 2. Comparison plots showing two option for Multiple parton interaction model

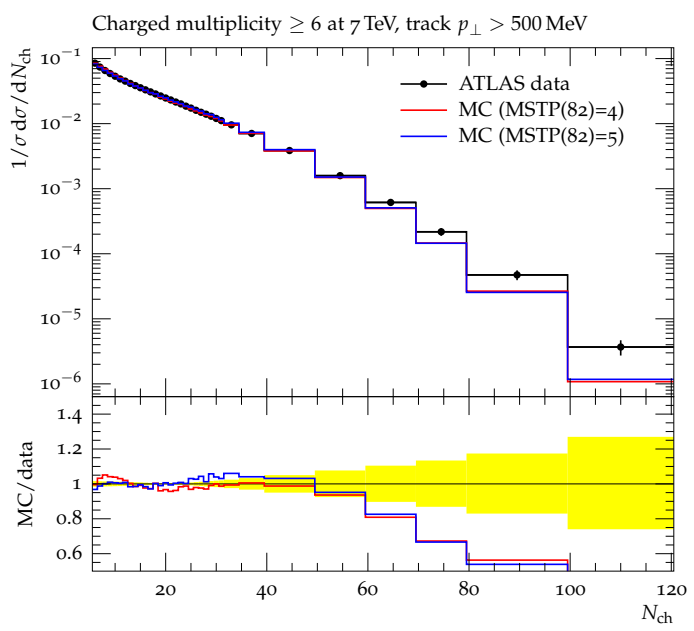

Figure 3. Comparison plots showing two options for Matter overlap

same $\Lambda_{Q C D}$ value is used both for the hard scattering and the initial and final state radiation.

- For $\operatorname{MSTP}(3)=1, \Lambda_{Q C D}$ values can be chosen by the user.

Multiplicity and Pt distributions show that model with single value of $\Lambda_{Q C D}$ is unable to describe data as shown in Figure 4. This fit is done using CTEQ5L PDF, so the value passed to the all three lambda is $0.192 \mathrm{GeV}$. Fit results show that to describe data well Lambda value for FSR is quite high around $0.5 \mathrm{Gev}$ where as value for $\mathrm{ME}$ is lower than $0.192 \mathrm{GeV}$. The Lambda for ISR is not sensitive to the MB data. Because of physics approximations used in event generator like PYTHIA6, it is not possible to describe data with the same value of QCD at different parts of the program.

\section{Matter Distribution}

The amount of scatterings is described by a matter overlap distribution between the two protons, which regulates how many central hard scatterings and how many less central softer scatterings happen. Two options of matter distribution are investigated with varying impact parameter approach.

- $\operatorname{MSTP}(82)=4$, hadronic matter overlap consistent with a double Gaussian matter distribution.

- $\operatorname{MSTP}(82)=5$, hadronic matter overlap consistent with an interpolation between a Gaussian and an exponential distribution.

This model effects shape of multiplicity and $\mathrm{Pt}$ as well shown in Figure 3. Double Gaussian describe data well for central eta values but for higher eta MC is well below the data opposite effect can be observed for other option. The approach which interpolates between Gaussian and exponential distribution looks favored by data as compared to double Gaussian approach.

\section{Color Reconnection}

The switch MSTP(95) gives control over selection of method for color reconnection in the final state. Three choices are studied to see their impact on data.

- $\operatorname{MSTP}(95)=0$, gives no color reconnection

- $\operatorname{MSTP}(95)=6$, gives the total probability for a string piece to survive the annealing and preserve its original color connections.

- $\operatorname{MSTP}(95)=8$, calculates the reconnection probability for each individual string piece by using the average string density in the region spanned by that string piece [5].

The pt distribution and average pt vs multiplicity (Nch), shown in Figure 5 appears to be sensitive to the color structure of the events, within the framework of the PYTHIA6 modeling. Pt distribution is badly described and average Pt shows quite soft spectrum when CR is switched off. It is clear from plots that to describe data CR should be switched on. The remaining two model choices describe data equally well except at the higher pt tail where the first choice MSTP(95)=6 seems better than other choice.

\section{Conclusion}

Different options of selected physics models offered by PYTHIA6 are investigated using best fit to ATLAS MB data at two energies. New scenario for MPI model should be switched on to get reasonable results for hadron hadron collision data. The MO option with an interpolation between a Gaussian and an exponential distribution is favored by data as compare to the double Gaussian distribution, though the difference is not much but visible at low PT and also at low multiplicity. Without the color reconnection model the Pt spectrum is too soft. The other two options for CR model do not show significant differences in the predictions. Monte Carlo simulations are unable to describe data with one $\Lambda_{Q C D}$ value for all parts of the event generation. To describe proton proton collision data at high energies, the model with adjustable $\Lambda_{Q C D}$ values 

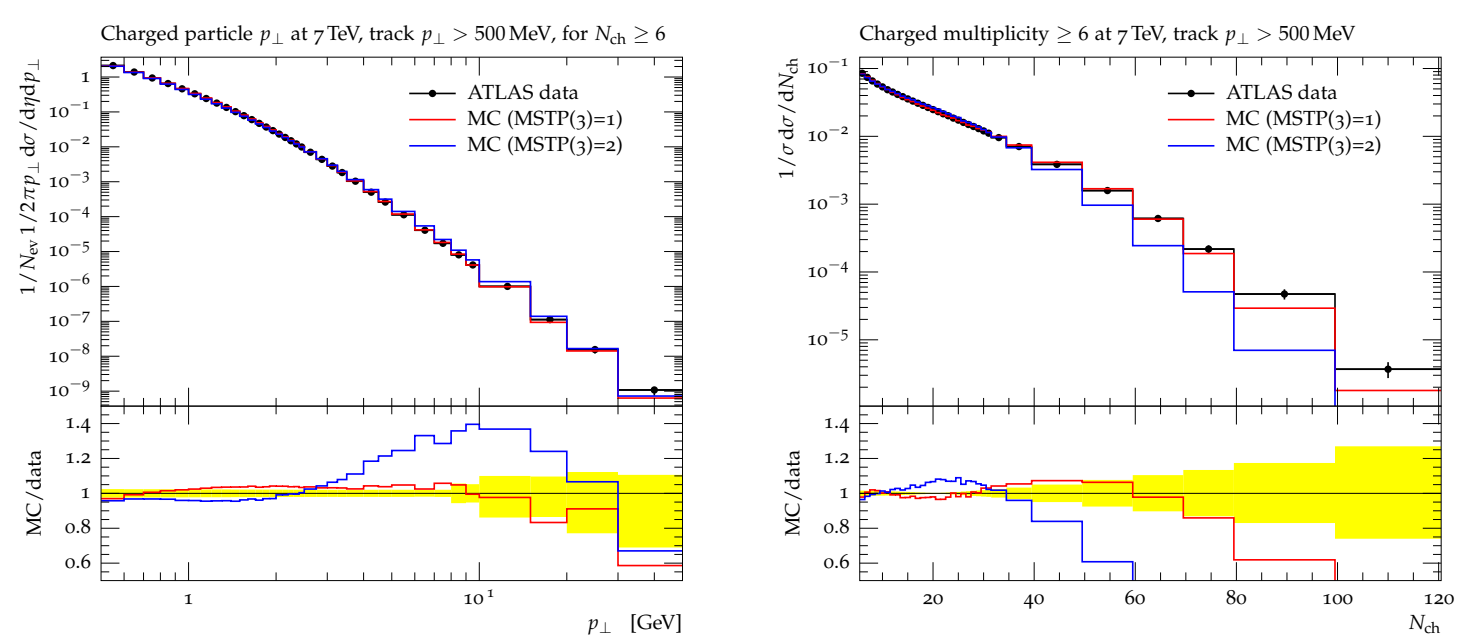

Figure 4. Comparison plots showing two options for $\Lambda_{Q C D}$ treatment
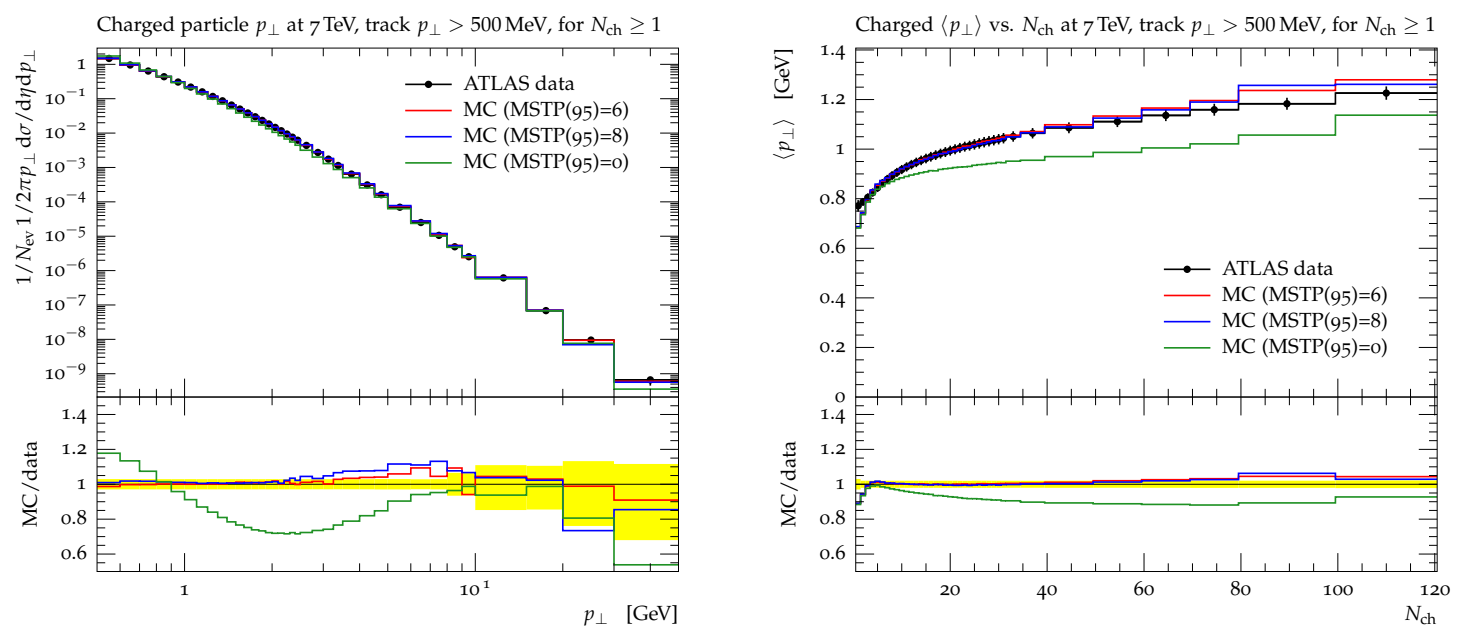

Figure 5. Comparison plots showing two options for color reconnections

should be used. All plots are produced using Rivet toolkit [4].

\section{References}

[1] Torbjörn Sjöstrand, Stephen Mrenna, and Peter Z. Skands. PYTHIA 6.4 Physics and Manual. JHEP, 05:026, 2006.

[2] R. Barate et al. Studies of quantum chromodynamics with the ALEPH detector. Phys.Rept., 294:1-165, 1998.
[3] G. Aad et al. Charged-particle multiplicities in pp interactions measured with the ATLAS detector at the LHC. New J.Phys., 13:053033, 2011.

[4] Andy Buckley, Jonathan Butterworth, Leif Lonnblad, Hendrik Hoeth, James Monk, et al. Rivet user manual. 2010.

[5] Peter Zeiler Skands. Tuning Monte Carlo Generators: The Perugia Tunes. Phys.Rev., D82:074018, 2010.

[6] Nameeqa Firdous and Gerald Rudolph. PYTHIA MPI model tuning to hadron collider data: Preliminary results. Nucl.Phys.Proc.Suppl., 207-208:73-76, 2010. 